\title{
On the Quadratic Assignment Problem
}

\section{INTRODUCTION}

THE BOUNDING part of the branch and bound algorithm is based on the solution of an assignment problem. The principles of such bound (when no facilities are already assigned) are described in [5]. Two strategies of employing the bound are tried. An improvement of this bound by using an algorithm by Murty [6] in which extreme points of the assignment problem are found in increasing order of the cost is also investigated. Although a substantial number of branches in the search are eliminated. the increase in the run time of each assignment problem seems to overshadow the gains.

Let $J$ be the set of $n$ sites, and $I$ be the set of the $n$ facilities to be located at these sites. The distance between sites $i$ and $j$ in $J$ is $d_{i j}$. The cost due to the distance between facilities $i$ and $j$ in $I$ is $c_{i j}$. Note that $d_{i j}=d_{i j}$. $d_{i i}=0, c_{i j}=c_{j i}, c_{i i}=0$. The problem is to find an assignment of facilities to sites (facility $i$ to be located at site $\left.i^{\prime}\right)$ such as to minimize the cost $F$ :

$$
F=\sum_{i, k} c_{i j} d_{i j}
$$

\section{THE ASSIGNMENT LOWER BOUND}

Assume that a set of facilities $I_{1}$, of cardinality $\alpha$, out of the set $I$ is assigned to sites in a set $J_{1}$, of the same cardinality $x$, out of the set $J$. The free facilities that belong to $I_{2}=I-I_{1}$ are yet to be assigned to the set of free sites $J_{2}=J-J_{1}$. In the following, a lower bound on the objective function $F$ for all possible assignments of free facilities to free sites is found.

The sum in equation (1) can be broken into three sums by the sets $I_{1}$ and $I_{2}$ as follows:

$$
\begin{aligned}
& F=\sum_{i \in l} \sum_{j i c l} c_{i j} d_{i j}
\end{aligned}
$$

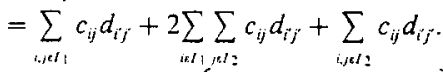

$$
\begin{aligned}
& F=\sum_{t, j e t \mid} c_{i j} d_{i j}+\sum_{j / t 2}\left\{2 \sum_{i t / 1} c_{i j} d_{i j}+\sum_{i / 2} c_{i j} d_{i j}\right\} \text {. } \\
& F=K+\sum_{r / 2} L_{i j^{\prime}}
\end{aligned}
$$

where

$$
\begin{aligned}
& K=\sum_{i, j i 1} c_{i j} d_{i j}
\end{aligned}
$$

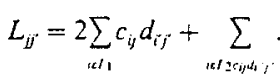

$K$ is constant for all the assignments of free facilities. When $j$ is located at a given site $j^{\prime} \varepsilon J_{2}$, the first sum in $L_{j,}$ is also constant. Since every facility must be assigned to a site, define a matrix $A=\left\{a_{r s}\right\}$ of dimensions $n-\alpha$ by $n-\alpha$ where:

$$
a_{r s}=\min \left\{L_{r s}\right\}=2 \sum_{i s / 1} c_{i r} d_{i s}+\min \left\{\sum_{i s / 2} c_{i r} d_{i s}\right\} .
$$

The minimum of the second sum in equation (4) is achieved when the $c_{i}$, are increasing and the $d_{i s}$ are decreasing. This result is proven in [3] (the proof there is wrong but the result is correct). For each $r$ we must choose one and only one $s$, and therefore, the assignment problem of minimizing $\sum_{r=1}^{n-x} a_{r s}$ for all possible assignments of $r$ 's to s's is a lower bound for the second sum of equation (2). Finally, add $K$ to the best assignment cost. and get a lower bound for all possible assignments of free facilities to free locations.

\section{THE BRANCH PROCEDURE}

There are many possibilities for the construction of the tree. One possibility is to go by order of locations i.e. first consider all possible facilities to be located on a certain site, for each of these facilities consider all possible facilities to be located on a second site, etc. Going by order of facilities is yet to be programmed and investigated. We can choose different orders of sites. In all test-problems the facilities are to be arranged in a rectangle. One way is to go from the center of the rectangle out, and the second way is to fix the corners of the rectangle first, and proceed inward. The second way has an advantage because we can use the symmetry of the problem and save $75 \%$ of the branches. The first four assignments are in the four corners of the rectangle and four permutations of each assignment are equivalent. This cannot be achieved in going by order of facilities. Let us call these two strategies $S_{1}$ and $S_{2}$ respectively.

\section{COMPUTATIONAL RESULTS}

The problem set given in [7] was used. Only the symmetric patterns were tested. The characteristics of the problems are summarized in Table 1.

Table 2 summarizes our results. The program is coded in FORTRAN IV on the Amdahl 5860 computer at the University of Michigan, Ann Arbor. It seems that run times behave like $(3.3)^{n} / 40,000$. If this estimate is correct, the 20 -facilities problem ought to be solved in about seven days.

\section{IMPROVED BOUNDS}

Murty [6] has proposed an efficient algorithm that finds the extreme solutions to an assignment problem in increasing values of the cost. The cost of the $N$ th best

Table 1. The problems

\begin{tabular}{rcc}
\hline Size & $\begin{array}{c}\text { Our lower } \\
\text { bound }\end{array}$ & $\begin{array}{c}\text { Best known } \\
\text { solution }\end{array}$ \\
\hline 6 & 41 & $43^{*}$ \\
8 & 93 & $107^{*}$ \\
12 & 247 & $289^{*}$ \\
15 & 482 & $575^{*}$ \\
20 & 1029 & $1287^{\circ}$ \\
30 & 2270 & $3078^{\circ}$ \\
\hline
\end{tabular}
Optimal solution. The optimality of the value 575
for the problem of size 15 is established here. ${ }^{\mathrm{a}}$ Found in [2].

${ }^{b}$ Found in $[4$, p. 210]. 
Table 2. Comparing two strategies

\begin{tabular}{|c|c|c|c|c|c|}
\hline \multirow[b]{2}{*}{ Size } & \multirow[b]{2}{*}{ Optimum } & \multicolumn{2}{|c|}{$S_{\mathrm{i}}$} & \multicolumn{2}{|c|}{$S$} \\
\hline & & $\begin{array}{l}\text { Run time } \\
\text { (sec) }\end{array}$ & $\begin{array}{l}\text { Assignment } \\
\text { problems }\end{array}$ & $\begin{array}{l}\text { Run time } \\
\text { (sec) }\end{array}$ & $\begin{array}{l}\text { Assignment } \\
\text { problems }\end{array}$ \\
\hline 6 & 43 & 0.041 & 58 & 0.063 & 32 \\
\hline 8 & 107 & 0.354 & 526 & 0.298 & 41 \\
\hline 12 & 289 & 78.492 & 42454 & +1.806 & 25790 \\
\hline 15 & 575 & - & - & 1518.928 & 520255 \\
\hline
\end{tabular}

-Was not attempted.

assignment is a lower bound for $F$ in equation (2) as long as it is not greater than the lowest value of $F$ among these $N$ assignments. Although this approach seems very promising, we could not yet find a way to get run times which are substantially better than those reported in Table 2. Using $N=24$, for example, and having four fewer levels in the tree, cuts substantially in the number of assignement problems solved (equivalent to the number of branches considered) but the solution time for each assignment problem increased and overshadowed the gain. Other strategies were also tried. The best strategy was to apply $N=24$ only when the first assignment is higher than $99 \%$ of the best solution (and of course less than $100 \%$ ). About $1 \%$ of the run time was saved. Different percentages were tried. but $99 \%$ was found to be the best for this set of problems.

Note that when a value of $F$, which is better than the best one found so far, is encountered in checking the $N$ quadratic assignments solutions, it can serve as a new best solution. Also, if the cost of the $K^{\prime}$ th assignment, $K \leq N$, is greater than or equal to the cost of the best solution found so far, we need not continue to find additional assignments.

\section{ACKNOWLEDGEMENTS}

I would like to thank Professor K Murty and $\mathrm{Mr}$ M Partovi of the Department of Industrial and Operations Engineering at the University of Michigan, Ann Arbor for providing the code of the assignment ranking program.

\section{REFERENCES}

1. Armour GC and Buffa ES (1963) A heuristic algorithm and simulation approach to relative location of facilities. Mgmt Sci. 9, 294-309.

2. Drezner $Z$ (1983) A practical approach to the facilitity layout problem. Submitted for Publication.

3. Gavett JW and Plyter NV (1966) The optimal assignment of facilities to locations by branch and bound. Ops Res. 14, 210-232.

4. Hitchings $G C$ and Cottam M (1976) An efficient heuristic procedure for solving the layout design problem. Omega 4, 205-214.

5. Lashkari RS and Jaisingh SC (1980) A heuristic approach to quadratic assignment problem. $J . O p t$ Res. Soc. 31, 845-850.

6. Murty KG (1968) An algorithm for ranking all the assignments in order of increasing cost. Ops Res. 16, 682-687.

7. Nugent CE, Vollmann TE and Ruml J (1968) An experimental comparison of techniques for the assignment of facilities to locations. Ops Res. 16, 150-173.

\section{Zvi Drezner}

School of Management

(Januar. 1984)

The University of Michigan-Dearborn

4901 Evergreen Road

Dearborn

Michigan 48128

USA 